\title{
OperA: Attention-Regularized Transformers for Surgical Phase Recognition
}

\author{
Tobias Czempiel ${ }^{1}$, Magdalini Paschali ${ }^{1}$, Daniel Ostler ${ }^{2}$, Seong Tae Kim ${ }^{1}$, \\ Benjamin Busam ${ }^{1}$, Nassir Navab ${ }^{1,3}$ \\ 1 Computer Aided Medical Procedures, Technische Universität München, Germany \\ 2 MITI, Klinikum Rechts der Isar, Technische Universität München, Germany \\ 3 Computer Aided Medical Procedures, Johns Hopkins University, Baltimore, USA
}

\begin{abstract}
In this paper we introduce OperA, a transformer-based model that accurately predicts surgical phases from long video sequences. A novel attention regularization loss encourages the model to focus on high-quality frames during training. Moreover, the attention weights are utilized to identify characteristic high attention frames for each surgical phase, which could further be used for surgery summarization. OperA is thoroughly evaluated on two datasets of laparoscopic cholecystectomy videos, outperforming various state-of-the-art temporal refinement approaches.
\end{abstract}

Keywords: Surgical Workflow Analysis - Surgical Phase Recognition . Transformers · Self-Attention · Cholecystectomy

\section{Introduction}

Surgical workflow analysis is a crucial task for the operating room (OR) of the future [1. Specifically, automatic detection of surgical phases is one of its most essential components. An efficient surgical phase recognition system will build the foundation for automated surgical assistance and cognitive guidance [2]. Online analysis during an ongoing intervention can provide feedback to surgeons and alarm the staff in case of erroneous or adverse events [4. Additionally, extracting surgical phases during an operation and group different procedures based on their unique characteristics plays an important role for modern surgical training. Since automatic extraction of surgical phases is particularly challenging, advanced Machine Learning (ML) methodologies [5] have been employed towards solving it. However, factors such as variability of patient anatomy, surgeon style [6] as well as limited availability and quality of training data present problems for modern ML algorithms.

A recent development in ML that could help overcome these challenges in surgical workflow analysis is transformer networks [7. Transformers have shown their vast potential for sequential modeling in Natural Language Processing (NLP) [8] and have quickly become the gold standard in this area. Transformer networks have the capability to create temporal relationships between current 
and previous frames using self-attention, much like frequently used LSTM methods 9 . However, self-attention enables learning in long sequences without forgetting of previous information which often hampers LSTM-based methods.

An additional advantage of transformer networks and self-attention over other approaches used for surgical phase recognition is their ability to visualize the attention weights for a sequence, which could yield further insights into the decision-making process of a model.

\section{$1.1 \quad$ Related Work}

Automatic extraction of surgical phases was initially performed using binary surgical signals [10, where a comparison with an average surgery determined the surgical phase. Hidden Markov Models (HMM), provided an extension of this idea capable of online predictions [11. In EndoNet, Twinanda et al. [5] utilized image features extracted with a Convolutional Neural Network (CNN) to predict the surgical phase and surgical tool presence directly from surgical images. EndoLSTM [12] additionally performed temporal refinement with LSTMs [9, which improved the results substantially. A variety of works combined pre-trained CNNs as feature extractors, followed by temporal refinement with LSTMs 1314. In MTRCNet-CL [15, Jin et al. proposed a CNN/LSTM model to refine the prediction over short sequences in an end-to-end fashion including a correlation loss to identify phase and tool correlations in an explicit manner. Czempiel et al. 16] proposed TeCNO, which combined Temporal Convolutional Networks (TCN) with a ResNet-50 17] feature extractor. Transformer models were first introduced for NLP [7] where they quickly became the state-of-the-art in a plethora of downstream tasks [18]8. Furthermore, the versatility of transformers has been showcased not only for vision tasks such as image classification [19] and text-to-image generation [20] but also in biology for the challenging protein folding problem with AlphaFold 21]. In surgical data sciences, transformers have been explored only for surgical tool 22] classification.

An additional aspect of transformers and self-attention is the fact that their attention weights could be used for model insights and explanation. Some works have claimed that attention has limited explanation capabilities 23. However, this assumption has been challenged 24] suggesting that each work should define their notion of explanation since it could be dependent on the task at hand.

In this paper, we introduce for the first time, OperA, a transformer-based method for online surgical phase prediction for laparoscopic operations. Our contributions are:

- We successfully leverage a transformer-based model for surgical phase recognition that outperforms other temporal refinement methods.

- We propose a novel attention regularizer, that improves the automatic extraction of the most relevant frames with high feature-quality.

- We utilize the attention weights to extract and visualize characteristic frames.

- We carefully evaluate OperA on two challenging surgical video datasets. 

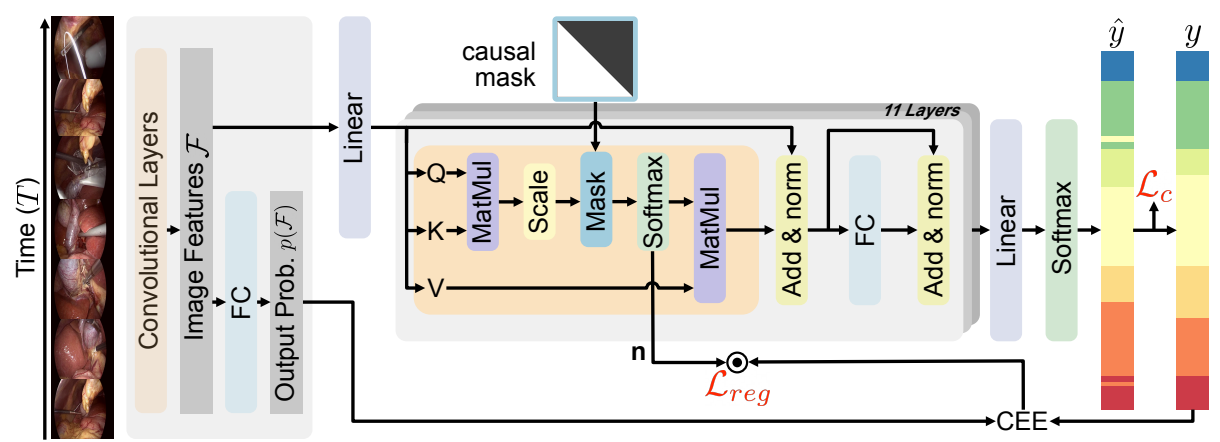

Fig. 1: Overview of the proposed OperA model. Image features $\mathcal{F}$ are used as input for the transformer. The output $\operatorname{logits} p(\mathcal{F})$ of the feature extraction backbone are used in combination with the normalized frame-wise attention weights $\mathbf{n}$ to regularize the attention.

\section{Methodology}

Our proposed model, OperA, consists of a CNN for visual feature extraction followed by multiple self-attention layers. The attention map is regularized during training to focus on reliable CNN image features. The full network architecture is visualized in Fig. 1 .

For our feature extraction backbone we trained a ResNet-50 [17] frame-wise CNN without sequential modeling. We trained this model on phase recognition and additionally on surgical tool detection, if tool information was available in the dataset. The result of the feature extraction backbone are per frame image features $\mathcal{F} \in \mathbb{R}^{2048}$ and their corresponding class probabilities $p(\mathcal{F}) \in[0,1]^{c}$ with $c$ the number of classes.

\subsection{Sequential Transformer Network}

Our model expands on the well-known Transformer architecture [7] with the addition of our attention regularization that will be discussed below. Transformers have the capabilities to model long sequences in a parallel manner using selfattention by relating every input feature with other input features regardless of their distance in the sequence [25]. Visualized in Fig. 11. we first calculate the query $Q$, key $K$ and value $V$, the inputs for the scaled dot product attention using a linear layer such that $(Q, K, V)=\operatorname{Linear}(\mathcal{F}) \in \mathbb{R}^{3 d}$ with $d=64$.

$$
\operatorname{AttentionWeights}(Q, K)=\operatorname{softmax}\left(\operatorname{mask}\left(\frac{Q K^{T}}{\sqrt{d}}\right)\right)
$$

$$
\operatorname{Attention}(Q, K, V)=\operatorname{AttentionWeights}(Q, K) V
$$


Our architecture uses 11 consecutive layers each consisting of a linear layer, a scaled dot-product attention layer, a layer normalization [26] and residual connections [17. Similar to the architecture of the Vision Tranformer [19] after the last encoding layer, a linear layer followed by a softmax is used to estimate the class-wise output probabilities $y$ for each frame of the sequence. For the training of the model we use a median frequency balanced cross-entropy loss 27 $\mathcal{L}_{c}$. Causal masking [28] with the binary mask $M \ni\{0,1\}$ is performed on the attention map of the model, to prevent information leakage from future frames to the current frame prediction. This allows us to use OperA for real-time surgical phase prediction.

\subsection{Normalized Frame-Wise Attention}

For each frame, each layer of our transformer generates one column in $A=$ AttentionWeights $(Q, K)$. Due to the softmax activation function (Eq. 1) each row of the attention map $A$ sums up to 1 . Column-wise summation of the attention weights in $A$ results in the total attention value for each frame at time $t$. Due to the causal mask visualized in Fig. 1 the first frame has the opportunity to contribute $T$ times, where $T$ is the number of frames in a video, while the last frame is considered only once. We therefore need to normalize the framewise attention by dividing the total attention of each frame with the number of times this frame is considered, thus equally weighting the attention of all frames regardless of their position in the video. The normalized frame-wise attention is calculated by: $\mathbf{n}=\left(n_{1}, \ldots, n_{T}\right)$ with $n_{j}=\frac{\sum_{i} A_{i j}}{\sum_{i} M_{i j}}$.

\subsection{Attention Regularization}

Different from NLP and Visual Transformers, the input of OperA is generated by a CNN backbone network. The quality of each frame embedding of the CNN can vary drastically, especially for frames where the CNN predictions are incorrect. To this end, OperA should focus on higher quality CNN features, that were correctly classified by the backbone CNN. Such features have higher softmax probabilities, or confidence, and lower cross-entropy values.

We learn this relationship by comparing the normalized frame-wise attention weights with the prediction error of our CNN. The regularization then reads:

$$
\mathcal{L}_{\text {reg }}=\langle\mathbf{n}, \operatorname{CEE}(p(\mathcal{F}), y)\rangle
$$

The Cross Entropy Evaluation value (CEE) describes the residual error of $p(\mathcal{F})$ compared to the ground truth label $y$. It should be noted that the weights of the backbone CNN remain frozen and CEE is only used for the optimization of the attention weights. Multiplying CEE with the normalized frame-wise attention $\mathbf{n}$ explicitly penalizes the model if a high attention value was generated for a feature with low CNN confidence. We apply the proposed regularization to the first attention layer as it has a direct relationship with the input visual features. The final loss function used for model training is denoted as: $\mathcal{L}=\mathcal{L}_{c}+\lambda \cdot \mathcal{L}_{\text {reg }}$ 
Summing up all the normalized attention weights for each layer we generate a final attention value for each frame. In order to interpret whether OperA focuses on highly-informative frames that correctly represent each phase, we extract the frames with Highest Attention (HA) and the ones with Lowest Attention (LA).

\section{Experimental Setup}

Datasets For the evaluation of OperA we use two challenging surgical workflow intra-operative video datasets of laparoscopic cholecystectomy procedures. The publicly available Cholec 80 [5] includes 80 videos with a resolutions of $1920 \times 1080$ or $854 \times 480$ pixels recorded at 25 frames-per-second (fps). For this work, the dataset was sub-sampled to 1fps. Every frame in the video has been manually assigned to one out of seven surgical phases. Additionally, seven different tool annotation labels sampled at $1 \mathrm{fps}$ are provided. We randomly select 20 videos for testing and the remaining 60 videos for training (48) and validation (12).

MitiSW (Miti Surgical Workflow) was collected and annotated by the MITI group at the Klinikum rechts der Isar in Munich. The dataset consists of 85 laparoscopic cholecystectomy videos with resolution $1920 \times 1080$ pixels and sampling rate of $1 \mathrm{fps}$. MitiSW includes the 7 surgical phases of Cholec 80 , shown in Fig. 3, along with one additional phase Pre-preparation, used to describe frames before the Preparation phase. The phases have been annotated by expert physicians with no additional tool-presence information. 20 videos are utilized for testing and the remaining 65 videos for training (52) and validation (13). For all the experiments 5 -fold cross validation is performed. To balance our combined loss function we set $\lambda$ to 1 .

Model Training OperA was trained for the task of surgical phase recognition using the Adam optimizer with an initial learning rate of 1e-5 for 30 epochs. We report the test results extracted by the model that performed best on the validation set for each fold. The batch size is identical to the length of each video. Our method was implemented in PyTorch and our models were trained on an NVIDIA Titan V 12GB GPU using Polyaxon The source code for OperA along with the Evaluation scripts is publicly available 5

Evaluation Metrics and Baselines To comprehensively measure the results we report the video-level Accuracy (Acc) the harmonic mean (F1) of Precision and Recall [11] and average the results over the 5 splits. We perform ablative testing to identify the most suitable number of attention layers and to test the effect of the regularization term (Sec. 2.3). Finally, we compare OperA with a variety of surgical phase recognition baselines.

\footnotetext{
${ }^{4}$ https://polyaxon.com/

${ }^{5}$ https://github.com/tobiascz/OperA/
} 
Table 1: Ablative testing results for 6 and 11 transformer layers and with the addition of Attention Regularization (Reg). Average metrics over 5 folds are reported (\%) with the corresponding standard deviation $( \pm)$.

\begin{tabular}{|c|c|c|c|c|c|}
\hline \multirow[b]{2}{*}{ Layers } & \multirow[b]{2}{*}{ Reg } & \multicolumn{2}{|c|}{ Cholec80 } & \multicolumn{2}{|c|}{ MitiSW } \\
\hline & & Acc & F1 & Acc & F1 \\
\hline 6 & - & $90.35 \pm 0.71$ & $83.45 \pm 0.32$ & $84.88 \pm 1.43$ & $84.92 \pm 1.12$ \\
\hline 6 & $\checkmark$ & $90.49 \pm($ & $.01 \pm$ & $85.41 \pm 1.26$ & $85.14 \pm$ \\
\hline 11 & - & $90.37 \pm 0.86$ & $83.85 \pm 0.33$ & $85.02 \pm 1.01$ & $85.28 \pm 0.98$ \\
\hline 11 & $\checkmark$ & $91.26 \pm 0.64$ & $84.49 \pm 0.60$ & $85.77 \pm 0.95$ & $85.44 \pm 0.79$ \\
\hline
\end{tabular}

\section{Results and Discussion}

Effect of Layers and Regularization In Table 1 we compare models trained with 6 and 11 attention layers and evaluate the impact of the attention regularization. 11 attention layers is the highest amount of layers that we could fit in the VRAM. For both datasets, the results slightly increase for 11 attention layers by $\sim 1 \%$ both in terms of Accuracy and F1-score. Regarding the attention regularization, a $\sim 1 \%$ improvement is reported for both datasets and number of layers. As we will discuss later, the attention regularization does not only marginally increases the model performance but also the quality of the highest attention video frames.

Baseline Comparison We compare OperA with various methods for surgical phase recognition. ResNet-50 is the feature extraction backbone, ResLSTM [14] and MTRCNet-CL [15] utilize LSTMs for the temporal refinement. Different to the other models MTRCNet-CL is trained in an end-to-end fashion combining a CNN feature extraction with LSTM training. One of the downsides to this approach is that due to memory constraint only a limited sequence of the video can be used per-batch. ResLSTM, TeCNO and OperA use pre-trained image features and can therefore analyze a full video sequence at once.

First, we see that temporal refinement achieves a substantial improvement over ResNet-50 ranging from $4-10 \%$ for Cholec 80 and 9-12\% for MitiSW, showcasing its advantage. MTRCNet-CL is outperformed by the other temporal models by $2-6 \%$ potentially potentially due to the limited sequence length that can be processed in every batch. OperA with or without positional encoding (PE) [7] outperforms the other temporal models by $2-6 \%$ in terms of accuracy for Cholec 80 showcasing the abilities of transformers to model long temporal dependencies. Regarding MitiSW, OperA without PE has increased accuracy by $0.6-3 \%$ and F1-score by $1 \%$ over all baselines. For both datasets it can be seen that PE marginally decreases the performance, potentially due to the increased sequence length in surgical videos in comparison to NLP tasks. 
Table 2: Baseline comparisons for Cholec 80 and MitiSW. MTRCNet-CL requires tool information, therefore cannot be used for MitiSW. We report the average metrics over 5 -fold cross validation along with their respective standard deviation $( \pm)$.

\begin{tabular}{cccccc} 
& \multicolumn{2}{c}{ Cholec80 } & \multicolumn{2}{c}{ MitiSW } \\
\cline { 2 - 3 } & Acc & F1 & & Acc & F1 \\
\cline { 2 - 3 } \cline { 5 - 6 } ResNet-50 & $81.21 \pm 1.16$ & $72.98 \pm 1.17$ & & $73.90 \pm 1.89$ & $71.53 \pm 1.41$ \\
ResLSTM & $87.94 \pm 0.80$ & $82.29 \pm 0.78$ & & $82.97 \pm 1.18$ & $84.06 \pm 1.15$ \\
MTRCNet-CL & $85.64 \pm 0.21$ & $80.94 \pm 0.95$ & & - & - \\
TeCNO & $89.05 \pm 0.79$ & $84.04 \pm 0.64$ & & $85.09 \pm 1.67$ & $84.18 \pm 1.53$ \\
OperA + PE & $90.20 \pm 1.45$ & $83.34 \pm 0.97$ & & $83.67 \pm 1.54$ & $84.04 \pm 1.20$ \\
OperA & $\mathbf{9 1 . 2 6} \pm \mathbf{0 . 6 4}$ & $\mathbf{8 4 . 4 9} \pm 0.64$ & & $\mathbf{8 5 . 7 7} \pm \mathbf{0 . 9 5}$ & $\mathbf{8 5 . 4 4} \pm \mathbf{0 . 7 9}$
\end{tabular}

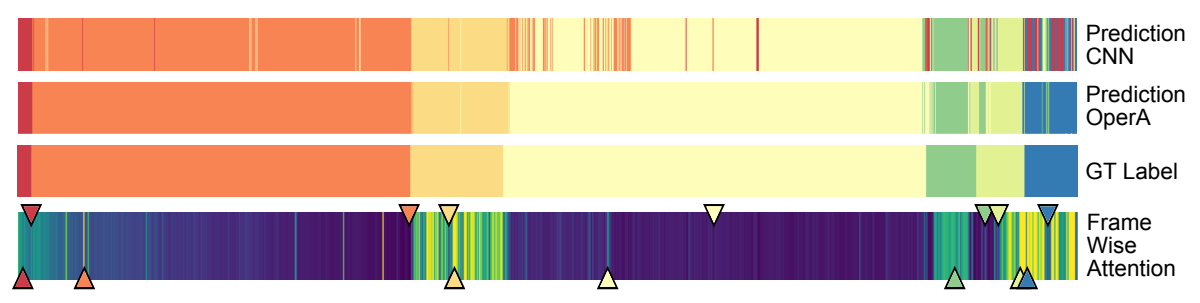

Fig. 2: Qualitative results of the predictions per phase for video 66 from Cholec80 using the feature extraction CNN and OperA compared to the ground truth labels. In the frame-wise attention, brighter (yellow) color corresponds to higher attention, darker (blue) color to lower attention. The position of the LA frames for each phase is denoted with $\nabla$ and the HA frames with $\triangle$.

Predictions and Attention Values In Fig. 2 we visualize the ground truth, predictions and attention values for video 66 of Cholec 80 . First, we see that the predictions of OperA are smoother and more consistent than the ones of the CNN. Moreover, the HA frames (denoted with $\triangle$ ) in most cases correspond with frames, where the CNN and OperA predictions were correct, while LA frames (denoted with $\nabla$ ) are at positions where the CNN predictions were wrong, confirming that attention regularization works as intended.

Highest and Lowest Attention Frames In Fig. 3 we visualize the HA and LA frames per phase for the models trained with and without attention regularization. Visual inspection revealed that LA frames are generally less descriptive for the respective surgical phase. However, as we can see highlighted by the blue boxes, the model trained without attention regularization has minimum attention for frames containing surgical tools that are quite characteristic of their respective phase. 


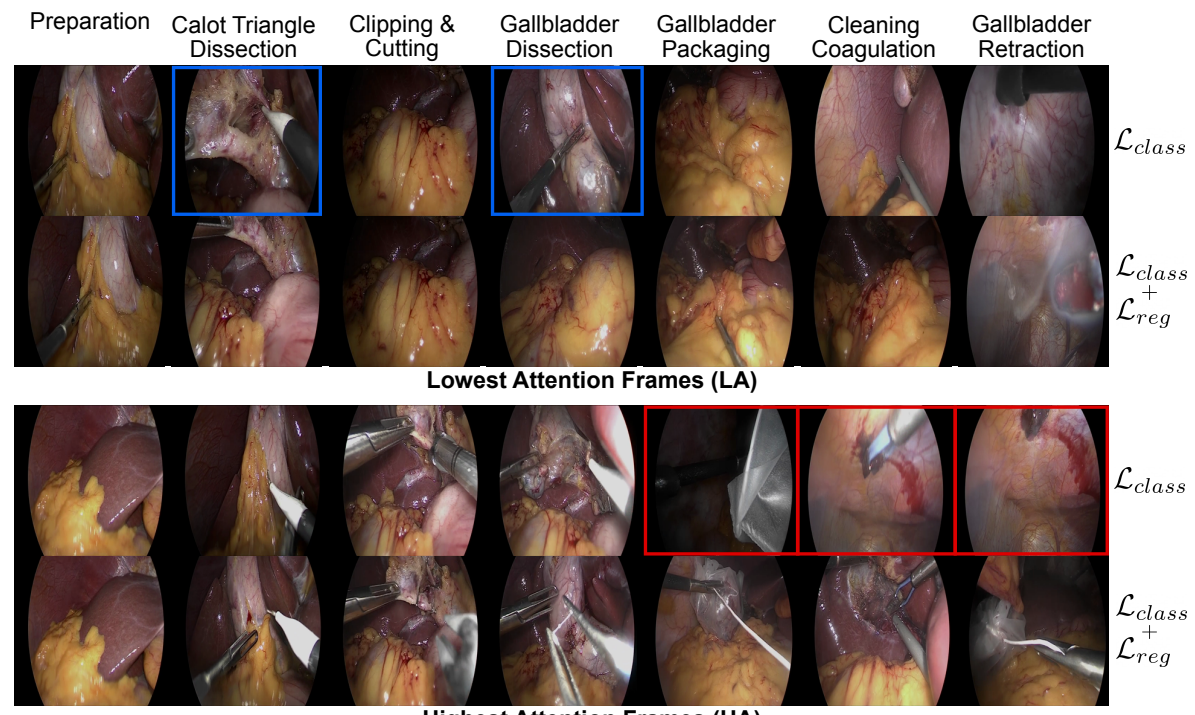

Highest Attention Frames (HA)

Fig. 3: Visualization of frames of video 66 of Cholec 80 with highest (HA) and lowest (LA) attention per phase for the models with and without attention regularization. Blue and red boxes denote frames of the model without regularization that have low attention, while they are descriptive of their phase and high attention, while they are not.

Regarding the HA frames, they are more diverse and representative of their surgical phase. For the model trained with attention regularization surgical tools are present in all phases besides "Preparation", highlighting the strong correlation between tools and surgical phases even though the attention model was not trained with tool information. With red boxes, we are showcasing the HA frames for the model trained without attention regularization. These frames were not descriptive of their phase and very similar to each other in the case of "Cleaning Coagulation" and "Gallbladder Retraction". These findings highlight the benefits of the proposed attention regularization and its potential for surgery video summarization.

\section{Conclusion}

In this paper we introduced OperA, a transformer-based model that accurately predicted surgical phases of cholecystectomy procedures, outperforming a variety of baselines on two challenging laparoscopic video datasets. Additionally, our novel attention regularizer enabled OperA to extract characteristic high attention frames. Future work includes applying our method to different laparoscopic procedures and explore its potential for surgical video summarization. 


\section{Acknowledgements}

This work is partially funded by the DFG research unit PLAFOKON (FKZ: NA620/33-2) and BMBF project ARTEKMED (FKZ: 16SV8088) in collaboration with the Minimal-invasive Interdisciplinary Intervention Group (MITI). Finally, we would like to thank NVIDIA for the GPU donation.

\section{References}

1. L. Maier-Hein, M. Eisenmann, C. Feldmann, H. Feussner, G. Forestier, S. Giannarou, B. Gibaud, G. D. Hager, M. Hashizume, D. Katic, et al., "Surgical data science: A consensus perspective," arXiv preprint arXiv:1806.03184, 2018.

2. C. R. Garrow, K.-F. Kowalewski, L. Li, M. Wagner, M. W. Schmidt, S. Engelhardt, D. A. Hashimoto, H. G. Kenngott, S. Bodenstedt, S. Speidel, et al., "Machine learning for surgical phase recognition: A systematic review," Annals of Surgery, 2020.

3. N. Padoy, "Machine and deep learning for workflow recognition during surgery," Minimally Invasive Therapy and Allied Technologies, vol. 28, pp. 82-90, 2019.

4. A. Huaulmé, P. Jannin, F. Reche, J. L. Faucheron, A. Moreau-Gaudry, and S. Voros, "Offline identification of surgical deviations in laparoscopic rectopexy," Artificial Intelligence in Medicine, vol. 104, no. May 2019, 2020.

5. A. P. Twinanda, S. Shehata, D. Mutter, J. Marescaux, M. De Mathelin, and N. Padoy, "EndoNet: A Deep Architecture for Recognition Tasks on Laparoscopic Videos," IEEE Transactions on Medical Imaging, vol. 36, no. 1, pp. 86-97, 2017.

6. I. Funke, S. T. Mees, J. Weitz, and S. Speidel, "Video-based surgical skill assessment using 3D convolutional neural networks," International Journal of Computer Assisted Radiology and Surgery, vol. 14, no. 7, pp. 1217-1225, 2019.

7. A. Vaswani, N. Shazeer, N. Parmar, J. Uszkoreit, L. Jones, A. N. Gomez, Ł. Kaiser, and I. Polosukhin, "Attention is all you need," Advances in Neural Information Processing Systems, vol. 2017-Decem, no. Nips, pp. 5999-6009, 2017.

8. J. Devlin, M. W. Chang, K. Lee, and K. Toutanova, "BERT: Pre-training of deep bidirectional transformers for language understanding," NAACL HLT 2019 - 2019 Conference of the North American Chapter of the Association for Computational Linguistics: Human Language Technologies - Proceedings of the Conference, vol. 1, no. Mlm, pp. 4171-4186, 2019.

9. S. Hochreiter and J. Schmidhuber, "Long Short-Term Memory," Neural Computation, vol. 9, no. 8, pp. 1735-1780, 1997.

10. S. A. Ahmadi, T. Sielhorst, R. Stauder, M. Horn, H. Feussner, and N. Navab, "Recovery of surgical workflow without explicit models," Lecture Notes in Computer Science (including subseries Lecture Notes in Artificial Intelligence and Lecture Notes in Bioinformatics), vol. 4190 LNCS, pp. 420-428, 2006.

11. N. Padoy, T. Blum, S. A. Ahmadi, H. Feussner, M. O. Berger, and N. Navab, "Statistical modeling and recognition of surgical workflow," Medical Image Analysis, vol. 16, no. 3, pp. 632-641, 2012.

12. A. P. Twinanda, N. Padoy, M. J. Troccaz, and G. Hager, "Vision-based Approaches for Surgical Activity Recognition Using Laparoscopic and RBGD Videos," Thesis, no. Umr 7357, 2017.

13. G. Yengera, D. Mutter, J. Marescaux, and N. Padoy, "Less is more: Surgical phase recognition with less annotations through self-supervised pre-training of cnn-lstm networks," arXiv preprint arXiv:1805.08569, 2018. 
14. Y. Jin, Q. Dou, H. Chen, L. Yu, J. Qin, C. W. Fu, and P. A. Heng, "SV-RCNet: Workflow recognition from surgical videos using recurrent convolutional network," IEEE Transactions on Medical Imaging, vol. 37, no. 5, pp. 1114-1126, 2018.

15. Y. Jin, H. Li, Q. Dou, H. Chen, J. Qin, C. W. Fu, and P. A. Heng, "Multi-task recurrent convolutional network with correlation loss for surgical video analysis," Medical Image Analysis, vol. 59, 2020.

16. T. Czempiel, M. Paschali, M. Keicher, W. Simson, H. Feussner, S. T. Kim, and N. Navab, "TeCNO: Surgical Phase Recognition with Multi-stage Temporal Convolutional Networks," in Medical Image Computing and Computer Assisted Intervention - MICCAI 2020, (Cham), pp. 343-352, Springer Publishing, 2020.

17. K. He, X. Zhang, S. Ren, and J. Sun, "Deep Residual Learning for Image Recognition," in 2016 IEEE Conference on Computer Vision and Pattern Recognition (CVPR), IEEE, jun 2016.

18. T. B. Brown, B. Mann, N. Ryder, M. Subbiah, J. Kaplan, P. Dhariwal, A. Neelakantan, P. Shyam, G. Sastry, A. Askell, S. Agarwal, A. Herbert-Voss, G. Krueger, T. Henighan, R. Child, A. Ramesh, D. M. Ziegler, J. Wu, C. Winter, C. Hesse, M. Chen, E. Sigler, M. Litwin, S. Gray, B. Chess, J. Clark, C. Berner, S. McCandlish, A. Radford, I. Sutskever, and D. Amodei, "Language models are few-shot learners," arXiv, 2020.

19. A. Dosovitskiy, L. Beyer, A. Kolesnikov, D. Weissenborn, X. Zhai, T. Unterthiner, M. Dehghani, M. Minderer, G. Heigold, S. Gelly, et al., "An image is worth 16x16 words: Transformers for image recognition at scale," arXiv preprint arXiv:2010.11929, 2020.

20. A. Ramesh, M. Pavlov, G. Goh, S. Gray, C. Voss, A. Radford, M. Chen, and I. Sutskever, "Zero-shot text-to-image generation," arXiv preprint arXiv:2102.12092, 2021.

21. L. Heo and M. Feig, "High-accuracy protein structures by combining machinelearning with physics-based refinement.," Proteins, vol. 88, pp. 637-642, may 2020.

22. S. Kondo, "Lapformer: surgical tool detection in laparoscopic surgical video using transformer architecture," Computer Methods in Biomechanics and Biomedical Engineering: Imaging \& Visualization, pp. 1-6, 2020.

23. S. Jain and B. C. Wallace, "Attention is not explanation," in Proceedings of the 2019 Conference of the North American Chapter of the Association for Computational Linguistics: Human Language Technologies, NAACL-HLT 2019, Minneapolis, MN, USA, June 2-7, 2019, Volume 1, pp. 3543-3556, Association for Computational Linguistics, 2019.

24. S. Wiegreffe and Y. Pinter, "Attention is not not explanation," in Proceedings of the 2019 Conference on Empirical Methods in Natural Language Processing and the 9th International Joint Conference on Natural Language Processing (EMNLPIJCNLP), (Hong Kong, China), pp. 11-20, Association for Computational Linguistics, Nov. 2019.

25. Y. Kim, C. Denton, L. Hoang, and A. M. Rush, "Structured attention networks," International Conference on Learning Representations, pp. 1-21, 2017.

26. J. L. Ba, J. R. Kiros, and G. E. Hinton, "Layer normalization," arXiv preprint arXiv:160\%.06450, 2016.

27. D. Eigen and R. Fergus, "Predicting Depth, Surface Normals and Semantic Labels with a Common Multi-scale Convolutional Architecture," in 2015 IEEE International Conference on Computer Vision (ICCV), IEEE, dec 2015.

28. R. Al-Rfou, D. Choe, N. Constant, M. Guo, and L. Jones, "Character-Level Language Modeling with Deeper Self-Attention," Proceedings of the AAAI Conference on Artificial Intelligence, vol. 33, jul 2019. 


\section{Supplementary}

(a) Ablative testing results for 6 and 11 transformer layers and with the addition of Attention Regularization (Reg). Average metrics over 5 folds are reported (\%) with the corresponding standard deviation $( \pm)$.

\begin{tabular}{|c|c|c|c|c|c|}
\hline Layers & Reg & Accuracy & $\mathrm{F} 1$ & Precision & Recall \\
\hline & & \multicolumn{4}{|c|}{ Cholec80 } \\
\hline 6 & - & $90.35 \pm 0.71$ & $83.45 \pm 0.32$ & $80.64 \pm 1.41$ & $86.48 \pm 0.61$ \\
\hline 6 & $\checkmark$ & $90.49 \pm 0.70$ & $84.01 \pm 0.39$ & $81.38 \pm 0.29$ & $86.98 \pm 0.61$ \\
\hline 11 & - & $90.37 \pm 0.86$ & $83.85 \pm 0.33$ & $81.60 \pm 0.40$ & $86.23 \pm 0.34$ \\
\hline \multirow[t]{2}{*}{11} & $\checkmark$ & $91.26 \pm 0.64$ & $84.48 \pm 0.60$ & $82.19 \pm 0.70$ & $86.92 \pm 0.86$ \\
\hline & & \multicolumn{4}{|c|}{ MitiSW } \\
\hline 6 & - & $84.88 \pm 1.43$ & $84.92 \pm 1.12$ & $82.76 \pm 1.43$ & $87.20 \pm 1.02$ \\
\hline 6 & $\checkmark$ & $85.41 \pm 0.95$ & $85.14 \pm 1.20$ & $83.00 \pm 1.34$ & $87.41 \pm 1.66$ \\
\hline 11 & - & $85.02 \pm 1.01$ & $85.28 \pm 0.98$ & $82.89 \pm 1.20$ & $87.82 \pm 0.75$ \\
\hline 11 & $\checkmark$ & $85.77 \pm 0.95$ & $85.44 \pm 0.78$ & $83.32 \pm 1.52$ & $87.68 \pm 1.08$ \\
\hline
\end{tabular}

(b) Baseline comparisons for Cholec80 and MitiSW. MTRCNET-CL requires tool information, therefore cannot be used for MitiSW. We report the average metrics over 5 -fold cross validation along with their respective standard deviation $( \pm)$.

\begin{tabular}{ccccc} 
& Accuracy & F1 & Precision & Recall \\
\hline & \multicolumn{4}{c}{ Cholec80 } \\
\cline { 2 - 5 } ResNet-50 & $81.21 \pm 1.16$ & $72.98 \pm 1.17$ & $68.35 \pm 1.61$ & $78.31 \pm 1.14$ \\
ResLSTM & $87.94 \pm 0.80$ & $82.29 \pm 0.78$ & $80.26 \pm 1.12$ & $84.43 \pm 0.85$ \\
MTRCNet-cl & $85.64 \pm 0.21$ & $80.94 \pm 0.95$ & $79.31 \pm 0.97$ & $82.67 \pm 0.114$ \\
TeCNO & $89.05 \pm 0.79$ & $84.04 \pm 0.64$ & $80.90 \pm 0.75$ & $\mathbf{8 7 . 4 4} \pm \mathbf{0 . 6 4}$ \\
OperA + PE & $90.20 \pm 1.45$ & $83.34 \pm 0.97$ & $80.78 \pm 1.42$ & $86.08 \pm 0.89$ \\
OperA & $\mathbf{9 1 . 2 6} \pm \mathbf{0 . 6 4}$ & $\mathbf{8 4 . 4 8} \pm \mathbf{0 . 6 0}$ & $\mathbf{8 2 . 1 9} \pm \mathbf{0 . 7 0}$ & $86.92 \pm 0.86$ \\
& & & & \\
& & MitiSW & \\
ResNet-50 & $73.90 \pm 1.89$ & $71.53 \pm 1.41$ & $69.06 \pm 1.38$ & $74.20 \pm 1.63$ \\
ResLSTM & $82.97 \pm 1.18$ & $84.06 \pm 1.15$ & $82.08 \pm 1.57$ & $86.15 \pm 0.94$ \\
TeCNO & $85.09 \pm 1.67$ & $84.18 \pm 1.53$ & $82.03 \pm 0.20$ & $86.50 \pm 0.43$ \\
OperA + PE & $83.67 \pm 1.54$ & $84.04 \pm 1.20$ & $81.34 \pm 1.60$ & $86.94 \pm 0.98$ \\
OperA & $\mathbf{8 5 . 7 7} \pm \mathbf{0 . 9 5}$ & $\mathbf{8 5 . 4 4} \pm \mathbf{0 . 7 9}$ & $\mathbf{8 3 . 3 2} \pm \mathbf{1 . 1 0}$ & $\mathbf{8 7 . 6 8} \pm \mathbf{0 . 7 1}$
\end{tabular}




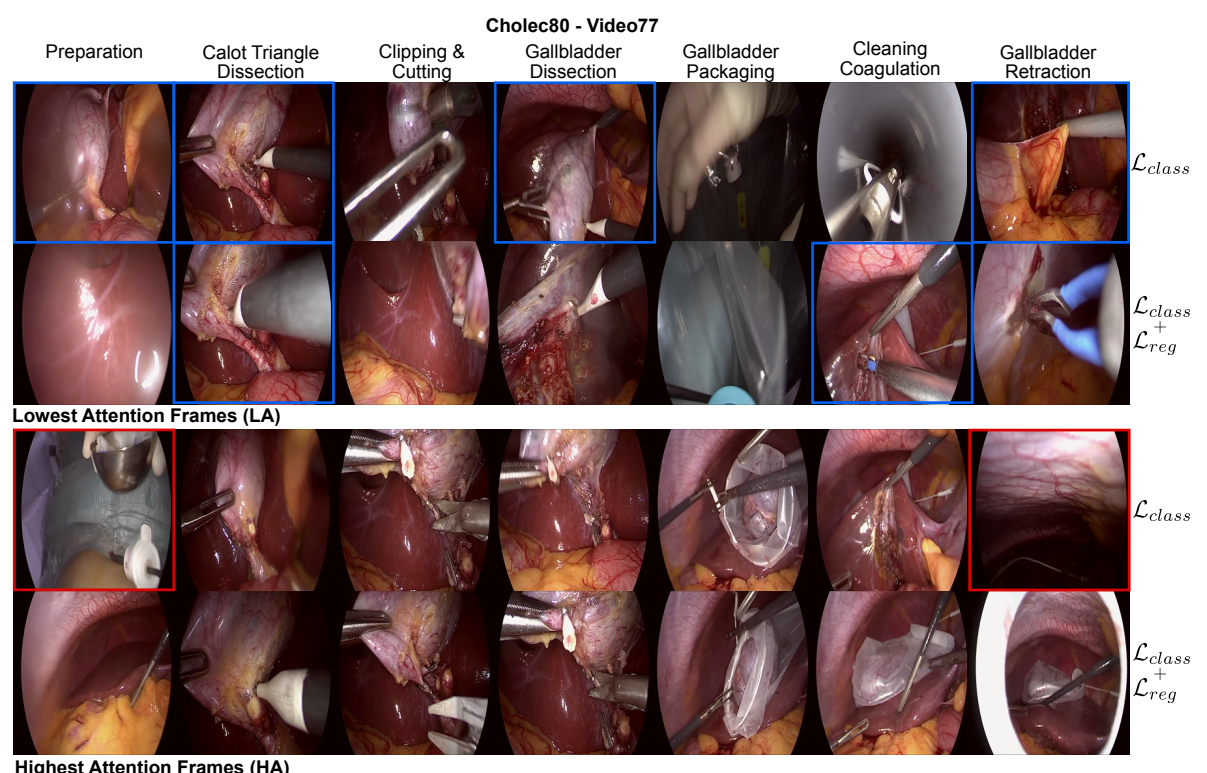

Highest Attention Frames (HA)
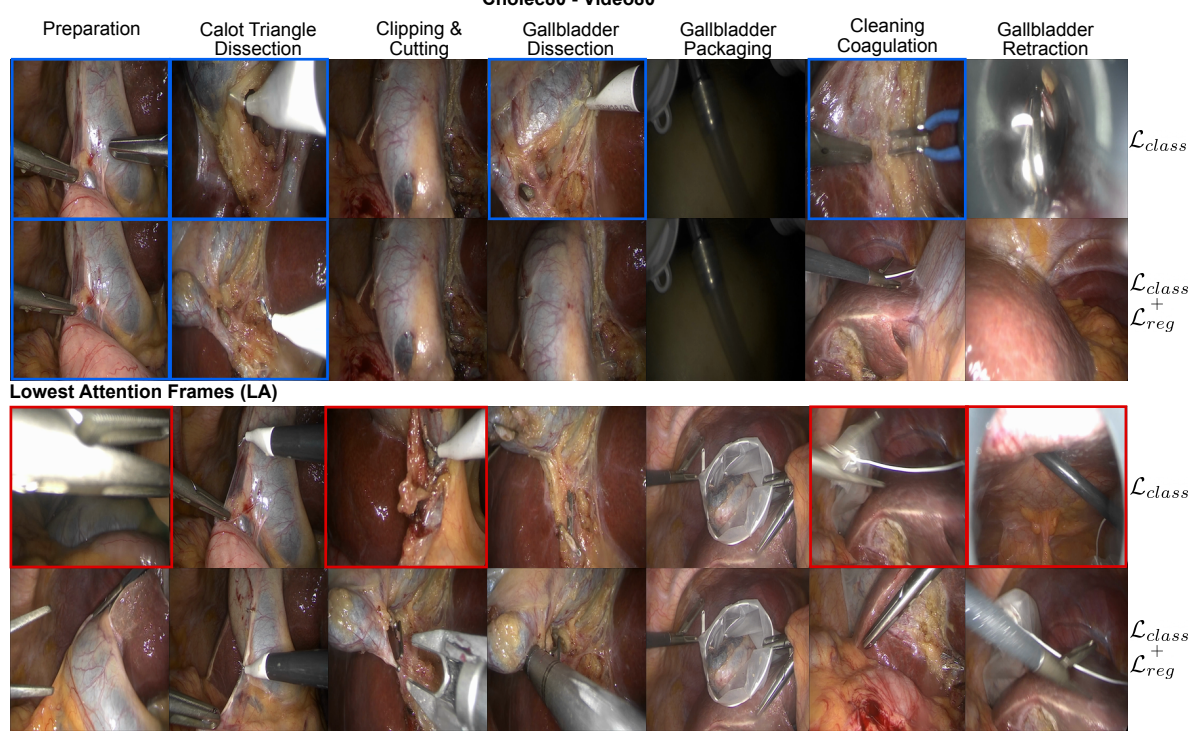

Highest Attention Frames (HA)

Fig. 4: Two more videos from Cholec 80 are visualized with maximum and minimum attention per phase for the models with and without attention regularization. Blue and red boxes denote frames of the model without regularization that have low attention, while they are descriptive of their phase and high attention, while they are not. 